\title{
Intérêt de la mutualisation des professionnels pour la planification d'activités médico-sociales
}

\section{Interest of the mutualisation of professionals when scheduling activities for Home Care and Services}

\author{
Alois Franzino ${ }^{1}$, Lorraine Trilling ${ }^{1}$, Maria Di Mascolo², Thibaud Monteiro \\ ${ }^{1}$ Univ Lyon, INSA Lyon, Université Claude Bernard Lyon 1, Univ Lumière Lyon 2, DISP, EA4570, 69621 Villeurbanne, \\ France, nom.prénom@insa-lyon.fr \\ ${ }^{2}$ Univ. Grenoble Alpes, CNRS, Grenoble INP, G-SCOP, 38000 Grenoble, France, maria.di-mascolo@grenoble-inp.fr
}

\begin{abstract}
RÉSUMÉ. Dans cet article, nous nous intéressons à l'organisation d'activités de soins à domicile. Nous nous concentrons sur la planification des accompagnements d'un ensemble d'enfants en situation de handicap par un SESSAD (Service d'Education Spéciale et de Soins A Domicile). Cette planification implique de décider conjointement de l'horaire de chaque prise en charge et du trajet des soignants. Pour organiser cette planification, il faut déterminer le professionnel en charge de chaque acte, où celui-ci se déroulera et comment organiser le planning pour réduire les frais de déplacement tout en maximisant la satisfaction des personnes recevant les soins. Un modèle de programmation linéaire en nombres entiers (PLNE) est proposé pour apporter une aide aux décideurs. Ce modèle permet, par une approche lexicographique, d'optimiser le temps de déplacement des professionnels, tout en gardant un temps d'attente réduit. L'analyse d'une étude de cas a permis d'étudier les besoins réels ainsi que les solutions qui peuvent être apportées et valide l'intérêt de mutualiser les professionnels pour planifier au mieux leurs tournées.

ABSTRACT. In this paper, we are interested in the organization of home care activities. We focus on the organization of the accompaniments of a set of people by a SESSAD (Service d'Education Spéciale et de Soins A Domicile - Special Education Service and Home Care). This organization implies deciding conjointly on the schedule of each care and on routing of the professional caregivers. To set this planning, we need to know who will do this work, where it will be done, and how to organize the schedule to reduce travel costs and to maximize the satisfaction of patients. A MILP is proposed to help decision-makers. This model allows, by a lexicographic approach, to optimize the travel time of professionals, while keeping a reduced waiting time. The analysis of a case study made it possible to study the real needs as well as the solutions that can be provided. Analysis validates the interest of pooling professionals to better plan their routes.

MOTS-CLÉS. Tournées de véhicule, Fenêtres de temps, Planification, Transport de personnel, Mutualisation.

KEYWORDS. Vehicle Routing Problem, Time windows, Scheduling, Employee Transportation, Sharing.
\end{abstract}

\section{Introduction}

En France, depuis une vingtaine d'années, les systèmes de santé sont en pleine mutation. Ces mutations ont pour objectif de profiter des évolutions technologiques et organisationnelles pour chercher une meilleure efficacité, conduisant à une amélioration des réponses aux besoins des patients, mais aussi une plus grande efficience, permettant une meilleure maîtrise des ressources mobilisées. Parmi les évolutions organisationnelles, il y a celle qui consiste à ne plus déplacer la personne à soigner vers la ressource de soin, mais à déplacer la ressource vers les personnes. Dans le secteur sanitaire, c'est le cas par exemple de l'hospitalisation à domicile (HAD) ou des services et soins infirmiers à domicile (SSIAD). Et dans ce secteur, les chercheurs du Génie Industriel se sont mobilisés pour améliorer et faciliter le fonctionnement de telles structures en cherchant notamment à augmenter le temps effectif de soin des personnels et à mâ̂triser les déplacements, générateurs de pertes de valeur ajoutée et de coûts financier et environnemental. Le secteur médico-social, quant à lui, n'a été que très peu étudié selon ce point de vue. Or, des structures comme les services d'éducation spécialisée et de soins à domicile (SESSAD) ou les services de soutien à l'éducation familiale et à l'intégration scolaire (SSEFIS) relèvent pleinement de la même problématique tout en ayant des spécificités qui méritent d'être explicitées. Deux spécificités seront particulièrement 
étudiées dans cet article : d'une part, la coordination des activités de soins et de celles du quotidien; et d'autre part, la gestion de l'espacement entre les activités de soins. La prise en charge par un SESSAD d'une personne en situation de handicap se fait sur un temps long, une ou plusieurs années. Pour garantir une meilleure accessibilité des soins et donc améliorer le résultat des prises en charge, il est impératif de coordonner les actions de soins avec les autres activités du quotidien. Ceci est nécessaire pour que ces soins ne soient pas vécus comme une interférence à la vie des patients.

Cet article s'intéresse à la planification des activités de soins et de services à domicile. Le champ d'analyse privilégié est celui du SESSAD. L'accompagnement d'un enfant en situation de handicap par un SESSAD peut inclure des actes médicaux spécialisés ainsi qu'une rééducation (kinésithérapie, orthophonie, psychomotricité, ergothérapie). Les éducateurs et enseignants spécialisés du SESSAD peuvent également apporter une assistance spécifique aux enfants en situation de handicap, soit sous forme de classe, soit individuellement, soit en petits groupes en dehors de la classe. Les accompagnements ont lieu dans plusieurs espaces de vie (domicile, école, etc.). Pour organiser ces accompagnements, il faut répondre à plusieurs questions : Qui réalise l'accompagnement (affectation)? Où aura lieu l'accompagnement (lieu)? Comment organiser au mieux les accompagnements pour réduire le nombre de trajets et maximiser la présence des professionnels auprès des usagers (tournée). Les travaux sont menés en lien étroit avec l'étude de terrain.

Le cas d'application concerne les visites des orthophonistes dans la région lyonnaise (France). Dans ce contexte, les enfants ayant besoin d'un traitement auditif peuvent bénéficier de ces soins soit directement sur le lieu scolaire, soit à leur domicile. La planification des tournées des professionnels est alors une tâche importante, au coeur de l'activité du service. Que ce soit au niveau des employés ou des patients, il faut considérer un nombre élevé de contraintes pour faire coïncider les emplois du temps de tous les acteurs. L'importance de l'optimisation des tournées est non seulement d'avoir l'avantage de réduire les coûts, mais aussi de pouvoir répondre au maximum à la demande.

Dans cet article, nous proposons une modélisation sous la forme d'un programme linéaire en nombres entiers mixtes pour résoudre ce problème de planification d'activités. Cet article est organisé comme suit : une revue de la littérature est donnée dans la section 2. La description de l'étude de cas et la formulation du problème sont présentées dans la section 3, suivie de la description du modèle mathématique dans la section 4. La section 5 contient les résultats de l'étude de cas avec la méthode exacte. Dans la section 6 on montre l'intérêt d'une démarche de clustering permettant de résoudre plus rapidement le problème complet. Des perspectives de recherche sont exposées dans la section 6.

\section{1. État de l'art et positionnement des travaux}

Depuis les années 2000, la planification des activités dans le secteur des soins et services à domicile a été étudiée sous de nombreux aspects, avec un intérêt croissant de la communauté scientifique, et l'on trouve de nombreuses références sur le sujet. Nous n'évoquerons ici que les publications les plus proches de notre problème. Pour compléter cet état de l'art succinct, nous recommandons la lecture des revues de littérature les plus récentes sur le sujet : (Cissé et al., 2017) analyse les travaux présents dans la littérature jusqu'en 2016 pour les problèmes de planification et de tournées dans le contexte des soins et services à domicile, dans le but d'identifier les paramètres communs à ces problèmes (objectifs, contraintes); (Grieco et al., 2020) s'intéresse plus généralement aux différents problèmes de décision rencontrés dans 
les structures de soins et services à domicile, et passe en revue les approches de la Recherche Opérationnelle et les méthodes de résolution utilisées pour aborder ces problèmes, par le biais d'une revue systématique de la littérature d'articles publiés jusqu'à fin septembre 2018. Plusieurs informations (objectif de l'étude, décisions modélisées, horizon de planification, approche de modélisation, approche de résolution...) sont extraites des articles analysés. Cette étude montre, entre autres, que, parmi les articles étudiés, la plupart (59 sur 77) sont consacrés au problème de planification; (Di Mascolo et al., 2021) présente une revue de littérature et une analyse bibliométrique de la littérature disponible à la mi mai 2019. Les articles analysés sont comparés, non seulement à partir des méthodes et outils de la RO utilisés, comme pour les revues précédentes, mais également à partir des caractéristiques réelles des problèmes résolus. Un ensemble de tableaux classifie les 153 articles étudiés, et fournit des informations détaillées sur chaque publication, les problèmes étudiés, les approches de modélisation et de résolution adoptées, les instances testées, les fonctions objectif étudiées, et les contraintes liées aux visites, aux patients et aux intervenants. Notons que l'un de ces tableaux se focalise sur les aspects incertains et dynamiques.

Ces revues de littérature nous permettent de remarquer qu'une grande variété de méthodes ont été développées pour résoudre le problème de la planification des soins et services à domicile. Le plus souvent, les auteurs proposent une résolution exacte, à travers un Programme Linéaire en Nombre Entiers (PLNE). Mais on observe un intérêt croissant pour des méthodes approchées, comme des heuristiques dédiées, ou des métaheuristiques, ou encore des méthodes hybrides, combinant plusieurs méthodes. Au niveau des critères d'optimisation, on peut constater que la minimisation des coûts liés au trajet est considérée dans pratiquement tous les articles étudiés. Nous pouvons également noter que la plupart des articles considèrent plusieurs critères d'optimisation, le plus souvent en les agrégeant dans une somme pondérée. Remarquons enfin que, ces dernières années, des critères liés aux préférences des patients et des soignants sont de plus en plus pris en compte. Pour ce qui concerne maintenant les contraintes prises en compte, on peut remarquer que la plupart des articles de la littérature considèrent des contraintes liées aux visites, classiquement rencontrées dans les problèmes de VRP (Vehicule Routing Problem), comme les fenêtres de temps, ou différents niveaux de qualification requis. Les contraintes liées aux intervenants sont un peu moins présentes et concernent essentiellement des fenêtres de temps de disponibilité, différents niveaux de qualification, ou des contraintes légales. On note cependant un certain nombre de contraintes liées aux patients, plus récentes et beaucoup moins nombreuses que les deux autres catégories évoquées ci-dessus. Elles concernent essentiellement des préférences des patients, à travers des fenêtres de temps souples et surtout la continuité de soins.

Dans le problème qui nous intéresse ici, nous cherchons à planifier sur un horizon d'une semaine, en optimisant deux objectifs. Le premier, la minimisation des temps de trajets, est, comme nous l'avons vu, un grand classique dans le domaine de la planification des soins et services à domicile. Le deuxième objectif, la minimisation des temps d'attente des intervenants, est présent uniquement dans $13 \%$ des articles cités dans (Di Mascolo et al., 2021), comme par exemple dans (Martinez et al. 2018) ou (Grenouilleau et al., 2019).

Nous prenons en compte plusieurs contraintes liées aux intervenants, toutes assez bien représentées dans la littérature : contraintes légales, à travers le nombre d'heures dans une journée de travail (Martinez et al. 2018), (Decerle, 2019), (Heching et al. 2019), fenêtres de temps strictes, pour représenter les disponibilités des intervenants (Martinez et al. 2018), (Decerle, 2019), (Heching et al. 2019), (Grenouilleau et al., 2019), (Tellez, et al. 2020), et plusieurs niveaux de qualification (Martinez et al. 2018), (Decerle, 2019), (Grenouilleau et al., 2019), (Heching et al. 2019). 
Nous prenons enfin en compte des contraintes liées aux visites. Certaines d'entre elles sont plutôt classiques, comme les contraintes temporelles avec des fenêtres de temps strictes (Martinez et al. 2018), (Decerle, 2019), (Heching et al. 2019), (Grenouilleau et al., 2019), (Tellez, et al. 2020), et les contraintes liées à un niveau de qualification requis (Martinez et al. 2018), (Decerle, 2019), (Grenouilleau et al., 2019), (Heching et al. 2019). Mais nous considérons également des contraintes beaucoup plus rarement prises en compte dans la littérature, comme des visites multiples sur l'horizon de planification (Martinez et al., 2018), (Alves et al., 2019), des patterns pour les visites, pour assurer un nombre de jours minimum et/ou maximum entre deux séances afin de favoriser l'efficacité des soins (Demirbilek et al., 2018) (Heching et al., 2019), et une contrainte, citée seulement par un seul (Demirbilek et al., 2018) des articles étudiés dans (Di Mascolo et al., 2021), qui est la possibilité de choisir parmi un ensemble de créneaux possibles. En effet, au lieu de considérer des données de disponibilité représentées classiquement par un "début" et une "fin", nous avons besoin de représenter ici les disponibilités des patients sur une journée par un ensemble de créneaux horaires potentiels, tous de la même durée. Cela permet d'avoir plusieurs périodes de disponibilité le même jour, et ainsi donner plus de flexibilité lors de l'élaboration du planning. Le service du jour n'utilisera qu'un seul créneau disponible sur une journée.

Pour conclure, nous constatons que dans le problème que nous traitons, nous retrouvons certaines caractéristiques déjà rencontrées dans la littérature, mais nous n'avons trouvé aucun article qui considère simultanément tous les aspects que nous avons à modéliser pour résoudre notre problème.

Notre contribution principale est celle de l'intégration, au sein d'un même modèle, des caractéristiques spécifiques du problème de planification des activités à domicile dans le contexte médico-social. Il s'agit notamment de proposer une prise en compte des créneaux de disponibilités des personnes prises en charge. Le second élément à relever est celui de la gestion de la distance temporelle entre activités. Ce travail nous a permis aussi de tester les limites d'une résolution exacte sur des cas réels, montrant la nécessité de : soit décomposer le problème complet par une démarche de clustering, soit d'utiliser des méthodes approchées.

\section{Description du problème}

\subsection{Description du service}

L'étude menée dans cet article est le fruit d'une collaboration avec un SESSAD de la Fondation OVE, association gestionnaire d'établissements et services médico-sociaux dans la région lyonnaise. Ce SES$\mathrm{SAD}$, dont une des catégories de professionnels est composée d'orthophonistes, ne dispose pas d'outil d'aide à la décision pour l'aider à établir les horaires de tournées des professionnels auprès des enfants accompagnés. Cette activité de planification, devant considérer un grand nombre de contraintes, est à la fois difficile à mener à la main et très chronophage. Il existe un réel potentiel d'amélioration sur cette activité de planification. Le modèle développé ici répond aux objectifs et aux contraintes de cette association et de ce SESSAD en particulier. L'objectif du modèle proposé est double : il s'agit, en priorité, de minimiser la durée de déplacement des collaborateurs, mais aussi, dans la mesure du possible, de minimiser le temps d'attente avant le démarrage d'une séance si le collaborateur arrive sur le lieu d'intervention avant le créneau horaire de disponibilité du patient.

Nous considérons un ensemble de patients nécessitant des interventions hebdomadaires d'un orthopho- 
niste en milieu scolaire (Une à 2 séances par semaine pour chaque patient). L'horizon considéré est celui de la semaine, à l'instar de l'horizon couvert par la résolution manuelle actuelle. Un pool de personnel est disponible pour répondre à la demande. Chaque professionnel a des qualifications différentes. Afin de répondre à une demande, un professionnel doit maîtriser les compétences nécessaires pour effectuer le service requis. En effet, les prestations ne sont pas toutes identiques et nécessitent certaines compétences spécifiques pour être honorées. Des fenêtres de temps strictes doivent être respectées afin de correspondre aux contraintes horaires des professionnels et des patients.

Sur la Figure 1, on peut voir la localisation des professionnels et des patients, et se rendre compte de l'éloignement des points à visiter et des points de départ des professionnels. Dans cet exemple, nous avons 12 orthophonistes (représentés par des étoiles grises) et 100 patients à visiter (représentés par des repères noirs, dont plusieurs se superposent lorsque les adresses sont proches ou que les patients fréquentent la même école).

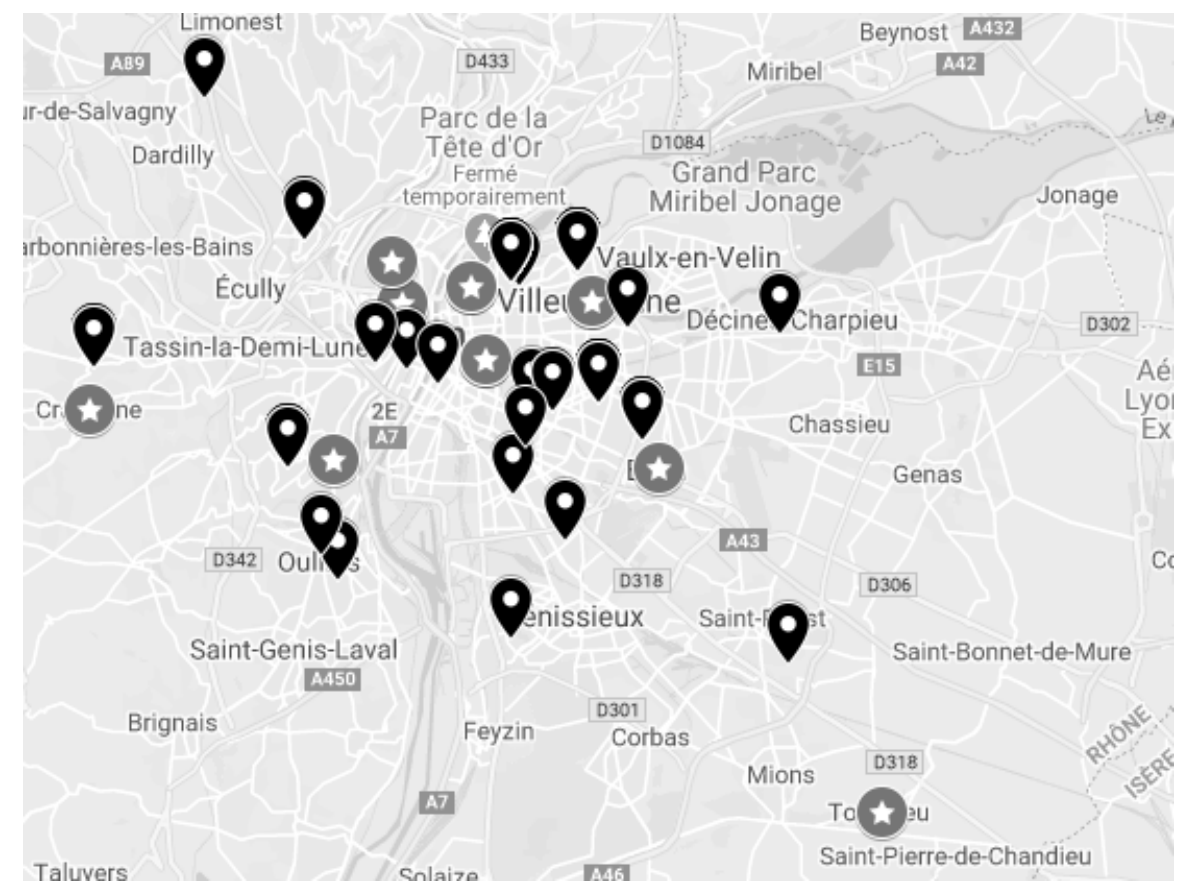

Figure 1.: Aperçu zoomé de la localisation des patients (repères noirs) et des orthophonistes (étoiles grises)

\subsection{Hypothèses retenues}

Ce problème de tournées et planification des activités de soins à domicile a é té modélisé comme un problème de tournées de véhicules avec fenêtre de temps (VRP-TW). Le problème est considéré comme un graphe $G(R, T)$ dans lequel les localisations des orthophonistes et les lieux de visite des patients sont des nœuds (R), et les chemins entre noeuds sont des arcs (T). Nous avons adopté une approche temporelle discrète dans laquelle le temps est divisé en tranches de temps afin de nous aligner avec les pratiques professionnelles. Chaque patient présente des besoins en soins, selon une fréquence donnée et requérant une compétence spécifique, qui peut être détenue par différents professionnels. Des créneaux de disponibilité sont définis pour chaque patient, une activité de soin ne pouvant être positionnée en dehors de ces créneaux. Une des spécificités du problème de planification des soins à domicile dans les SESSAD, qui est de garantir un nombre de jours minimum entre deux séances, est prise en compte. La résolution se faisant à l'échelle de la semaine, nous avons choisi de ne pas intégrer un nombre maximum 
entre deux séances.

Les professionnels n'ont pas tous les mêmes contrats de travail : ils n'ont donc pas les mêmes capacités de temps de travail, que ce soit en nombre de jours dans la semaine ou en nombre d'heures de travail par jour. Nous partons de l'hypothèse que le respect des temps de travail des employés est à l'échelle journalière et hebdomadaire.

Notre objectif est de trouver une adéquation entre les horaires des professionnels et des patients, tout en nous assurant que l'employé destiné à un certain patient possède les bonnes compétences. Il est également à noter que chaque employé quitte son domicile au début de la tournée et doit terminer celle-ci à la maison, à l'intérieur de la plage de travail définie dans son contrat.

Nous considérons plusieurs objectifs dans notre formulation mathématique. L'objectif est en priorité de minimiser le temps de trajet des professionnels. Dans un deuxième temps on cherche à minimiser le temps d'attente d'un professionnel sur le lieu du service, entre son arrivée et le début du service. Ces deux objectifs sont combinés dans une approche lexicographique, qui nous permet de les hiérarchiser et donc de garantir un temps de trajet minimal.

\section{Modèle mathématique}

Le problème est modélisé sous la forme d'un programme linéaire en nombre entiers (PLNE), dans lequel des variables binaires et des variables entières positives sont utilisées. Par sa structure, le modèle de décision fait partie des problèmes NP difficiles.

Les notations du modèle sont résumées dans le tableau 3.1. On nommera localisation l'union des points de départ des professionnels au début de la journée et des lieux de soins des patients. Chaque localisation est relative à une personne (professionnel ou patient) et constitue un sommet du réseau à organiser. Les localisations correspondant aux patients sont caractérisées par des demandes pour certains services $\left(D e m_{p s}=1\right)$. Les localisations (patients ou professionnels) sont disponibles uniquement sur certains créneaux horaires en fonction du temps de travail des professionnels et des contraintes d'emploi du temps des patients $\left(A v a i_{r d w}=1\right)$. Les professionnels sont qualifiés pour la réalisation de certains services uniquement $\left(Q E_{e s=1}\right)$. Une marge temporelle (MTime) par rapport au début du traitement est tolérée, cela signifie que le professionnel peut arriver légèrement en avance ou en retard par rapport à la date de début planifiée.

\section{Variables}

- $X_{r_{1} r_{2} e d w}$ : égale à 1 si l'employé $e$ réalise le trajet de $r_{1}$ à $r_{2}$ le jour $d$ sur le binaire créneau $w$

- $Y_{\text {erd }}: \quad$ horaire d'arrivée de l'employé $e$ au point $r$ le jour $d$ entier positif

- $T f_{e d}$ : $\quad$ horaire de fin de la journée de travail de l'employé $e$ le jour $d$ entier positif

\section{Objectifs}




\begin{tabular}{|c|l|}
\hline \multicolumn{2}{|c|}{ Données } \\
\hline \hline Notation & Définition \\
\hline$P$ & Ensemble des lieux de traitement des patients \\
\hline$E$ & Ensemble des points de départ des professionnels/employés \\
\hline$R=E \cup P$ & Ensemble des localisations à prendre en compte \\
\hline$D$ & Ensemble des jours \\
\hline$S$ & Ensemble des services \\
\hline$W$ & Ensemble des créneaux \\
\hline$T_{r_{1} r_{2}}$ & Temps de trajet entre les localisations $r_{1}$ et $r_{2}$ \\
\hline Dem$_{p s}$ & $\begin{array}{l}\text { Requête du patient } p \\
=1 \text { si la localisation } p \text { requiert le service } s,=0 \text { sinon }\end{array}$ \\
\hline Avai ${ }_{r d w}$ & $\begin{array}{l}\text { Disponibilité de la localisation } r \text { (patient ou professionnel) } \\
=1 \text { si la localisation } r \text { est disponible le jour } d \text { sur le créneau } w,=0 \\
\text { sinon }\end{array}$ \\
\hline$Q E_{e s}$ & $\begin{array}{l}\text { Qualification de l'employé } e \\
=1 \text { si l'employé } e \text { est qualifié pour réaliser le service } s,=0 \text { sinon }\end{array}$ \\
\hline Max $D_{e}$ & Nombre d'heures de travail maximum par jour pour l'employé $e$ \\
\hline$M a x H_{e}$ & Nombre d'heures de travail maximum par semaine pour l'employé $e$ \\
\hline$T$ Time & Temps de traitement \\
\hline WTime & Longueur d'un créneau \\
\hline MTime & $\begin{array}{l}\text { Marge temporelle entre l'arrivée et le début du traitement } \\
\text { (avance ou retard du professionnel toléré par rapport au début du } \\
\text { traitement) }\end{array}$ \\
\hline$K_{p}$ & $\begin{array}{l}\text { Nombre minimum jours demandés entre chaque traitement pour le } \\
\text { patient localisé en } p\end{array}$ \\
\hline Horizon de temps \\
\hline$M$ & grande valeur supposée infinie, appelée Grand M \\
\hline
\end{tabular}

Tableau 3.1.: Notation du modèle

OptTrav $=\min \sum_{r_{1} \in R} \sum_{r_{2} \in R} \sum_{e \in E} \sum_{d \in D} \sum_{w \in W} X_{r_{1} r_{2} e d w} * T_{r_{1} r_{2}}$

OptWaitT $=\min \sum_{e \in E} \sum_{d \in D}\left(T f_{e d}-Y_{e e d}\right)-\sum_{r \in R} \sum_{s \in S} D_{e m} * T$ Time $-\sum_{r_{1} \in R} \sum_{r_{2} \in R} \sum_{e \in E} \sum_{d \in D} \sum_{w \in W} X_{r_{1} r_{2} e d w} * T_{r_{1} r_{2}}$

\section{Initialisation des variables}

$$
X_{e_{1} r e_{2} d w}=0 \quad \forall e_{1} \in E, r \in R e_{2} \in E / e_{2} \neq e_{1}, d \in D, w \in W
$$


$X_{\text {rredw }}=0 \quad \forall e \in E, r \in R, d \in D, w \in W$

$X_{r p e d w} \leq A v a i_{p d w} * A v a i_{e d w} \quad \forall e \in E, d \in D, w \in W: w \neq 0, r \in R, p \in P$

$X_{\text {eped } 0} \leq A v a i_{p d 0} * A v a i_{e d 0} \quad \forall e \in E, d \in D, p \in P$

\section{Contraintes}

$\sum_{r_{2} \in R} \sum_{e \in E} \sum_{w \in W} X_{r_{2} r_{1} e d w} \leq 1 \quad \forall r_{1} \in P, d \in D$

$\sum_{r \in R} \sum_{w \in W} X_{e r e d w} \leq 1 \quad \forall e \in E, d \in D$

$\sum_{r \in R} \sum_{w \in W} X_{r e e d w} \leq 1 \quad \forall e \in E, d \in D$

$\sum_{r_{1} \in R} \sum_{r_{2} \in R} X_{r_{1} r_{2} e d w} \leq 1 \quad \forall e \in E, d \in D, w \in W$

$Y_{p_{1} e d}+$ TTime $+T_{p_{1} p_{2}} \leq Y_{e p_{2} d}+M * \sum_{w \in W}\left(1-X_{p_{1} p_{2} e d w}\right) \quad \forall e \in E, p_{1} \in P, p_{2} \in P, d \in D$

$\left.Y_{\text {eed }}+T_{e p} \leq Y_{\text {epd }}+M *\left(1-\sum_{w \in W} X_{\text {epedw }}\right)\right) \quad \forall e \in E, p \in P, d \in D$ 
$Y_{e p d}-T_{e p} \leq Y_{e e d}+M *\left(1-\sum_{w \in W} X_{e p e d w}\right) \quad \forall e \in E, p \in P, d \in D$

$\left(w * \sum_{r \in R} X_{r p e d w} * W T i m e\right)-M T i m e \leq Y_{e p d} \quad \forall e \in E, p \in P, d \in D, w \in W$

$Y_{e p d} \leq M\left(1-\sum_{r \in R} X_{r p e d w}\right)+\sum_{r \in R} X_{r p e d w} * w * W T i m e+$ MTime $\quad \forall e \in E, p \in P, d \in D, w \in W$

$Y_{e r_{1} d} \leq M * \sum_{r_{2} \in R} \sum_{w \in W} X_{r_{2} r_{1} e d w} \quad \forall e \in E, r_{1} \in R, d \in D$

$\sum_{e \in E} \sum_{r \in R} \sum_{d \in D} \sum_{w \in W}\left(X_{r p e d w} * Q E_{p s} * A v a i_{e d w} * A v a i_{p d w}\right) \geq D e m_{p s} \quad \forall p \in P, s \in S$

$\sum_{r_{2} \in R} \sum_{w \in W} X_{r_{2} r_{1} e d w}=\sum_{r_{2} \in R} \sum_{w \in W} X_{r_{1} r_{2} e d w} \quad \forall e \in E, r_{1} \in R, d \in D$

$M *\left(1-\sum_{r \in R} \sum_{w_{2}=w}^{|W|-1} X_{\text {pred }}\right) \leq M *\left(1-\sum_{r \in R} X_{r p e d w}\right) \quad \forall e \in E, p \in P, d \in D, w \in W$

$\sum_{r_{2} \in R: r_{2} \neq r_{1}} \sum_{e \in E} \sum_{f=d}^{d+K_{r_{1}}} \sum_{w \in W} X_{r_{1} r_{2} e f w} \leq 1 \quad \forall r_{1} \in P, d \in\left[0, \ldots,|D|-K_{r_{1}}\right]$

$\sum_{r \in R} \sum_{e \in E} \sum_{d \in D} \sum_{w \in W} X_{r p e d w}=\sum_{s \in S} D_{e} m_{p s}$

$$
Y_{\text {erd }}+\sum_{w \in W}\left(X_{\text {reedw }} *\left(T_{r e}+\text { TTime }\right)\right) \leq T f_{e d} \quad \forall e \in E, r \in P, d \in D
$$




$$
\begin{aligned}
& \sum_{r \in R} \sum_{d \in D} \sum_{w \in W} X_{r p e d w} \leq M * \sum_{s \in S}\left(Q E_{e s} * D e m_{p s}\right) \quad \forall p \in P, e \in E \\
& T f_{e d}-Y_{e e d} \leq M a x D_{e} \forall e \in E, d \in D \\
& \sum_{d \in D} T f_{e d}-Y_{e e d} \leq M a x W_{e} \forall e \in E \\
& X_{r_{1} r_{2} e d} \in\{0,1\} \quad \forall r_{1} \in R, r_{2} \in R, e \in E, d \in D \\
& Y_{e r_{1} d} \in \mathbb{N} \quad \forall r_{1} \in R, e \in E, d \in D \\
& T f_{e d} \in \mathbb{N} \quad \forall e \in E, d \in D \\
& v_{e} \in \mathbb{N} \quad \forall e \in E
\end{aligned}
$$

Le premier objectif (1) minimise la somme des temps de trajet de l'ensemble des tournées des professionnels sur l'horizon de la planification. Le deuxième objectif formulé en (2) minimise le cumul des temps d'attente de l'ensemble des professionnels sur l'horizon. Ce temps d'attente est calculé à partir de la durée totale des journées des professionnels, à laquelle on retranche les temps de traitements auprès des patients ainsi que les temps de transport (calculés pour le premier objectif).

Les contraintes (3) à (7) correspondent à un pré-processing initial visant à donner la valeur 0 à toutes les variables qui le nécessitent. Un employé ne peut pas partir du domicile d'un autre employé (3). Un employé ne peut pas avoir comme destination le domicile d'un autre employé (4). Un trajet relie un point de départ et d'arrivée qui sont différents (5). Un employé ne peut réaliser un soin auprès d'un patient que si les deux sont disponibles sur le créneau (6). Les contraintes (7) expriment la même chose sur le premier créneau avec un départ du dépôt (soit le domicile du professionnel).

Les contraintes (8) à (11) sont relatives à la formulation classique d'un problème de tournées de véhicules. La contrainte (8) interdit de réaliser plusieurs visites chez le même patient le même jour, (9) et (10) contrôlent le nombre de départs et d'arrivées à partir des noeuds "professionnels". La contrainte (11) interdit à l'employé de visiter plus d'un patient en même temps.

Le respect de la fenêtre temporelle est formulé par les contraintes (12) à (17). La continuité des heures de passages pendant la tournée d'un professionnel est gérée par les contraintes (12). Les contraintes (13) et (14) contrôlent l'heure de départ des professionnels. Les contraintes (15) et (16) imposent les heures d'arrivée auprès des patients, qui doit se situer au début du soin, avec une tolérance de plus ou moins la marge temporelle. Les contraintes (17) font le lien entre la réalisation d'un trajet entre deux localisations et l'heure d'arrivée de ce trajet. 
La compatibilité de disponibilité entre les patients et les employés est donnée par les contraintes (6) et (7). La contrainte (18) vérifie que la demande est satisfaite avec les bonnes compétences des employés. La conservation du flux est exprimée à l'aide des contraintes (19) et (20). Les patients doivent disposer d'un certain nombre de jours entre deux séances successives, cela se traduit par la contrainte (21). La contrainte (22) garantit que le nombre de visites effectuées est égal à la demande totale. L'heure de fin de travail de l'employé est déterminée par la contrainte (23). Le fait qu'un employé ne peut visiter un patient que s'il possède la compétence requise est exprimé par la contrainte (24).

Les contraintes (25) et (26) traduisent le respect du temps de travail maximum à l'échelle de la journée et de la semaine pour chacun des professionnels.

Les contraintes (27), (28), (29) et (30) déterminent les zones de définitions de variables.

\section{Expérimentation}

\subsection{Approche de résolution}

Pour cette étude, nous utilisons le solveur CPLEX 12.8.0 pour résoudre notre modèle linéaire en nombre mixte.

Il est important de déterminer la valeur du grand $\mathrm{M}$ utilisé dans ces expérimentations. Le grand $\mathrm{M}$ est relatif au nombre maximum de créneaux à prendre en compte dans l'horizon de décision et du nombre de localisation du problème. Il peut être évalué grâce à la formule suivante :

$$
M=\max \sum_{r \in R} \sum_{d \in D} \sum_{w \in W}\left(\text { Avai }_{r d w} * w * \text { WTime }\right) .
$$

Le temps d'attente doit être optimisé, afin d'éviter de générer des planning avec des horaires inappropriés, incluant de longs temps d'attente avant les séances. Nous adoptons une approche lexicographique : Lexmin (OptTravel, OptWaitT). Pour ce faire, après avoir trouvé le temps de parcours optimal à l'aide du premier objectif (1), nous ajoutons une contrainte sur le temps de parcours, et utilisons la minimisation du temps d'attente (équation 2) comme objectif. Pour accélérer la résolution et pour une meilleure exploitation du temps de calcul, la solution trouvée lors de l'optimisation de l'objectif 1 est utilisée comme solution initiale de la seconde phase d'optimisation.

\subsection{Instances}

À l'aide des données de la fondation OVE, nous avons généré des instances qui prennent en compte les horaires actuels des orthophonistes, la durée moyenne d'un traitement (45 min), la demande des patients ainsi que leur taux de disponibilité.

Les valeurs des temps de trajet ont été calculées grâce à des requêtes réalisées sur le site d'OSRM (Open Source Routing Machine, http ://project-osrm.org/) à partir des coordonnées GPS des établissements scolaires des patients et des domiciles de professionnels. Nous avons analysé des emplois du temps d'écoliers, de collégiens et de lycéens, pour élaborer les disponibilités des patients, en ciblant les plages libres. La valeur de $K_{p}$ (nombre de jours minimum entre deux séances) est prise aléatoirement, soit 0 soit 1 . 
Par absence de données fiables sur les quotités de travail des professionnels, nous avons choisi de relaxer les contraintes liées au respect du temps de travail journalier et hebdomadaire, en partant du principe que les matrices de disponibilités des patients limitent intrinsèquement l'amplitude des journées de travail.

\begin{tabular}{|c|c|}
\hline Nombre d'orthophonistes & $|E|=12$ \\
\hline Nombre de patients & $|P|=100$ \\
\hline Horizon de temps & $|D|=5$ \\
\hline Nombre de services & $|S|=3$ \\
\hline Matrice de temps de trajet & $T_{r_{1} r_{2}}$ \\
\hline Matrice de la demande & Dem $_{p s}$ \\
\hline Matrice de disponibilité & Avai $i_{r d w}$ \\
\hline Temps de traitement & TTime $=45$ \\
\hline Durée d'un créneau & WTime $=60$ \\
\hline Marge de temps avant traitement & MTime $=5$ \\
\hline Matrice de temps entre 2 traitements & $K_{p}$ \\
\hline Grand M & $M$ \\
\hline
\end{tabular}

Tableau 4.2.: Données de l'instance globale

Devant la taille des instances, nous avons décidé de mener les expérimentations sur différentes sousinstances afin d'évaluer le comportement du modèle. A titre d'exemple, un pool de 10 orthophonistes amène à un modèle contenant plus de 3,5 millions de variables binaires et plus de 6 millions de contraintes. Chaque sous-instance testée comprend un ensemble d'orthophonistes (sous-ensemble de E) ainsi qu'un ensemble de patients (sous-ensemble de P) qui leur étaient auparavant affectés par la fondation OVE.

Des tests sont d'abord menés séparément sur 3 sous-instances contenant chacune un seul orthophoniste (instance mono-employé), puis nous combinons ces 3 sous-instances afin d'évaluer les gains de temps de transport quand les problèmes sont résolus dans une approche intégrée. Il n'y a alors plus de préaffectation d'un orthophoniste à un patient, les décisions d'allocation et de tournées doivent être prises en même temps. Nous analysons ensuite ces résultats en les associant à d'autres critères d'évaluation des solutions, tels que le temps de travail et le temps d'attente.

Les instances contenant plusieurs orthophonistes sont basées sur les mêmes paramètres d'entrée que les instances mono-employé (disponibilité, localisation, temps de trajet, ...).

Dans le tableau 4.3 , nous pouvons observer les performances pour les instances mono-employé, avec les orthophonistes A, B et C. Cette approche, qui sépare la décision pour chaque employé, est assez conforme aux pratiques actuelles adoptées par les gestionnaires du SESSAD. Les patients sont en effet déjà affectés à des professionnels. Ceci à la fois dans un souci de continuité des traitements d'une séance sur l'autre, mais aussi pour simplifier le problème de l'organisation des activités qui est faite manuellement par le gestionnaire. Ces affectations, correspondant au fonctionnement actuel, ont été établies par la fondation OVE sur la base des compétences et des compatibilités de profils, mais aussi des souhaits des orthophonistes. Elles sont donc cohérentes, du point de vue de la pratique métier, mais peuvent certainement être améliorées d'un point de vue efficacité.

On retrouve dans le tableau 4.4 les résultats de l'approche intégrée rassemblant 3 employés (et leurs 
27 patients), en comparaison aux résultats cumulés du tableau 4.3 . Nous constatons ici une diminution du temps de trajet, du temps de travail et du temps d'attente pour l'approche intégrée. Notons également que les temps de travail et temps d'attente obtenus pour l'approche intégrée $(\mathrm{A}, \mathrm{B}, \mathrm{C})$ lorsqu'on ne fait que minimiser le temps de trajet, sont respectivement de 2848 et 917 . L'approche lexicographique nous donne ainsi un gain de 5\% sur le temps travaillé et $15 \%$ sur le temps d'attente.

\begin{tabular}{|c|c|c|c|c|c|}
\hline Employé & $\begin{array}{c}\text { Nombre } \\
\text { de patients }\end{array}$ & $\begin{array}{c}\text { Nombre } \\
\text { de visites }\end{array}$ & $\begin{array}{c}\text { Temps de } \\
\text { transport }\end{array}$ & $\begin{array}{c}\text { Temps de } \\
\text { travail }\end{array}$ & $\begin{array}{c}\text { Temps } \\
\text { d'attente }\end{array}$ \\
\hline \hline $\mathrm{A}$ & 9 & 15 & 125 & 1223 & 423 \\
\hline $\mathrm{B}$ & 10 & 12 & 174 & 1196 & 482 \\
\hline $\mathrm{C}$ & 8 & 10 & 89 & 733 & 194 \\
\hline
\end{tabular}

Tableau 4.3.: Performance des instances mono-employé A,B and C

\begin{tabular}{|c|c|c|c|}
\hline $\begin{array}{c}\text { SLT's } \\
\text { Instance }\end{array}$ & $\begin{array}{c}\text { Temps de } \\
\text { transport }\end{array}$ & $\begin{array}{c}\text { Temps de } \\
\text { travail }\end{array}$ & $\begin{array}{c}\text { Temps } \\
\text { d'attente }\end{array}$ \\
\hline \hline $\begin{array}{c}\text { Approche } \\
\text { séparée } \\
\text { A+B+C }\end{array}$ & 388 & 3152 & 1099 \\
\hline $\begin{array}{c}\text { Approche } \\
\text { intégrée } \\
\text { (A,B,C) }\end{array}$ & 266 & 2707 & 776 \\
\hline $\begin{array}{c}\text { Gain de la } \\
\text { solution } \\
\text { intégrée }\end{array}$ & $31 \%$ & $14 \%$ & $29 \%$ \\
\hline
\end{tabular}

Tableau 4.4.: Comparaison des performances pour les employés A, B et C

\subsection{Minimisation du temps de trajet}

Les données réelles comprennent un ensemble de 12 orthophonistes pour un total de 100 patients. Nous évaluons le gain de temps de trajet d'une instance (OptTrav) en le comparant à la somme des temps de trajet des orthophonistes travaillant individuellement avec leurs propres patients (SumTrav). Le gain est exprimé par un taux de diminution et calculé comme suit : 1-OptTrav/SumTrav.

Nous considérons 7 catégories d'instances, allant de 3 à 9 orthophonistes. Pour chaque catégorie d'instances, 5 tests sont réalisés à partir de données différentes.

Les résultats sont donnés dans le tableau 4.5 et la figure 2 . On peut observer que le gain en temps de parcours augmente presque linéairement avec le nombre d'employés regroupés dans la décision globale. Cependant, le temps de compilation augmente de façon exponentielle lorsque le nombre d'employés dépasse 6 (Figure 3) . Il semble donc peu raisonnable de chercher à exécuter le programme sur le pool complet des 12 orthophonistes en même temps. Il s'agit alors de contourner la difficulté de résolution liée à la taille de l'équipe. Ayant un temps de calcul réduit avec 6 orthophonistes et un gain de temps de trajet linéaire, il nous semble judicieux de diviser les 12 employés en deux groupes de 6 , c'est ce que nous verrons dans la section 5 . 


\begin{tabular}{|c|c|c|}
\hline $\begin{array}{c}\text { Nombre } \\
\text { d'employés }\end{array}$ & $\begin{array}{c}\text { Taux moyen de diminution } \\
\text { du temps de trajet (\%) }\end{array}$ & $\begin{array}{c}\text { Temps d'exécution } \\
\text { moyen (min) }\end{array}$ \\
\hline \hline 3 & $22 \%$ & 1 \\
\hline 4 & $28 \%$ & 1 \\
\hline 5 & $29 \%$ & 1,6 \\
\hline 6 & $35 \%$ & 4,4 \\
\hline 7 & $37 \%$ & 21,2 \\
\hline 8 & $39 \%$ & 101,2 \\
\hline 9 & $41 \%$ & 299,6 \\
\hline
\end{tabular}

Tableau 4.5.: Performance avec objectif de diminution du temps de trajet

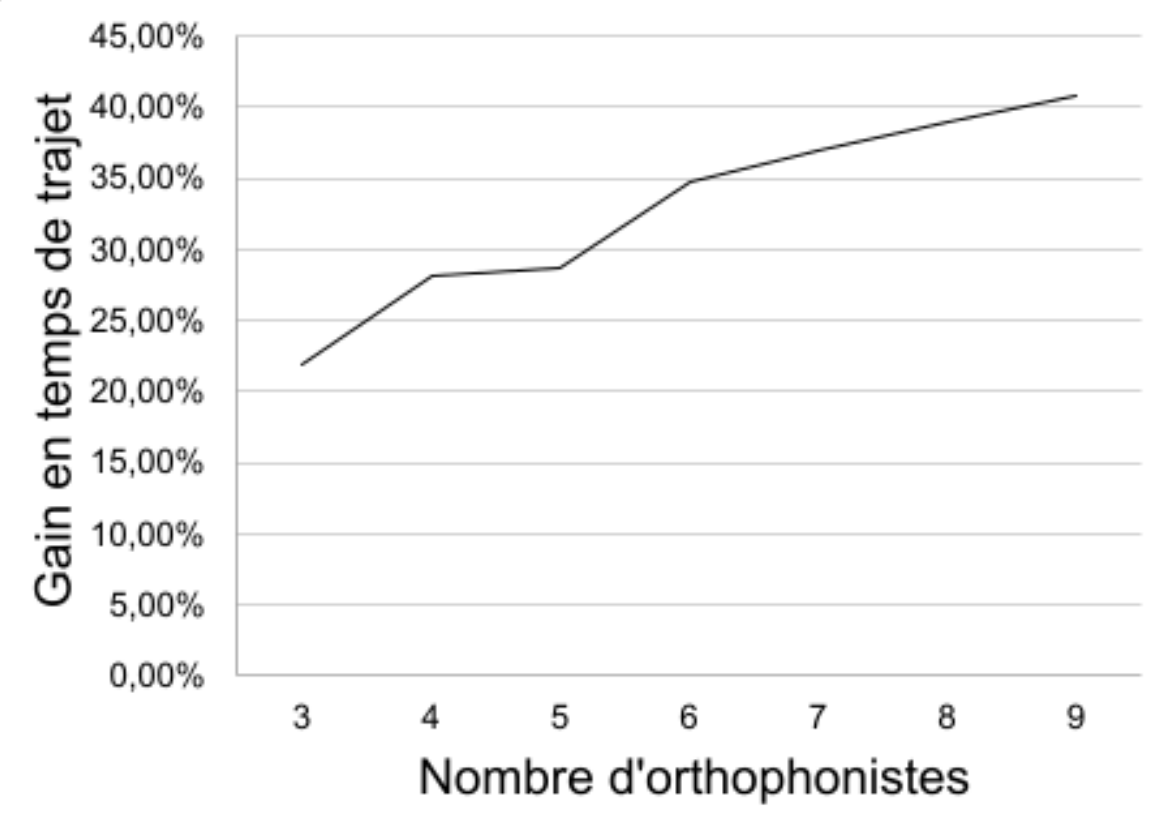

Figure 2.: Diminution du temps de trajet selon le nombre d'employés

\subsection{Minimisation du temps de travail}

Comme pour le premier objectif, la réduction des temps de travail est obtenue en comparant le temps trouvé par le modèle PLNE, que l'on notera $(O p t W o r k T)$ et la somme des temps individuels notée $(S u m W o r k T)$. Le taux de diminution du temps de travail est alors donné par la formule : 1 OptWorkT/SumWorkT. Il en est de même pour le temps d'attente.

Ces résultats ont été recueillis après avoir exécuté le modèle sur les deux objectifs, de manière lexicographique, comme présenté dans la section 5. Notons que, par rapport à la seule minimisation du temps de trajet, l'approche lexicographique apporte un gain de $24 \%$ en moyenne sur le temps d'attente pour les exemples testés, allant même jusqu'à $43 \%$, et un gain moyen de $7 \%$ pour le temps de travail, avec un gain $\max$ de $12 \%$.

Comme on peut l'observer dans le tableau 4.6 , seuls les résultats des instances de 3 à 6 employés sont présentés. Cela est dû au temps de calcul qui est encore plus long quand on optimise de manière lexicographique en résolvant à nouveau le modèle PLNE avec le second objectif. 


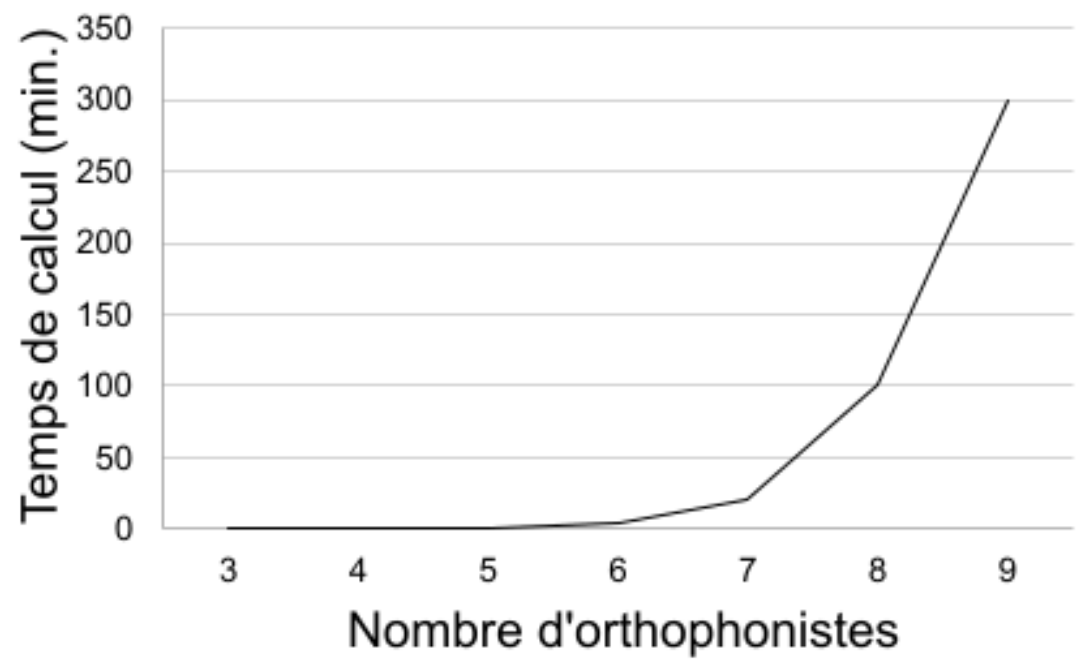

Figure 3.: Temps d'exécution selon le nombre d'employés

Nous pouvons voir que nous réduisons non seulement le temps de trajet, mais également le temps de travail et le temps d'attente. On ne trouve pas de tendance aussi marquée que celle observée sur le temps de trajet, mais on peut dire que l'approche intégrée autour d'un pool d'employés nous permet de gagner sur tous les aspects présentés.

Nous avons également examiné les tendances des résultats en prenant les résultats non plus en considérant le nombre d'orthophonistes mais le nombre de patients ou de demandes de service. Ces expérimentations n'ont rien montré de concluant, ce qui nous a conduit à retenir les résultats en partant du nombre d'employés.

\begin{tabular}{|c|c|c|}
\hline $\begin{array}{c}\text { Nombre } \\
\text { d'employés }\end{array}$ & $\begin{array}{c}\text { Taux moyen de diminution } \\
\text { du temps de travail }\end{array}$ & $\begin{array}{c}\text { Taux moyen de réduction } \\
\text { du temps d'attente }\end{array}$ \\
\hline \hline 3 & $7 \%$ & $14 \%$ \\
\hline 4 & $6 \%$ & $7 \%$ \\
\hline 5 & $5 \%$ & $7 \%$ \\
\hline 6 & $5 \%$ & $6 \%$ \\
\hline
\end{tabular}

Tableau 4.6.: Performance avec objectif de diminution du temps d'attente

\subsection{Conclusion sur le modèle exact}

Cette étude a pu montrer l'intérêt potentiel de l'utilisation d'une approche intégrée considérant un pool d'employés pour la prise en charge des demandes de traitement par les patients, par rapport à l'approche séparée, qui est en place aujourd'hui et dans laquelle les orthophonistes ont une liste prédéfinie de patients affectés. Les expérimentations ont montré que les temps de parcours peuvent être réduits significativement avec l'approche intégrée. Les performances augmentent en fonction de la taille de l'instance (nombre d'orthophonistes). Le problème comprenant 5 dimensions, les temps de calcul explosent lorsque le nombre d'employés dépasse 6 , il est donc recommandé d'utiliser ce programme pour un maximum de 6 employés. Une solution pour surmonter cette difficulté d'explosion combinatoire est d'utiliser un algorithme de clustering séparant le pool d'orthophonistes en 2 clusters de 6 employés au maximum. 
Cette idée est explorée dans la section suivante du document.

\section{Vers une démarche de clustering}

Devant l'explosion combinatoire des temps de calcul nécessaires à trouver une solution de qualité pour les instances d'une taille similaire à la réalité de terrain, nous abordons la question de la complexité de cette résolution. Nous proposons de diviser la taille de l'instance afin de traiter des sous-ensembles plus petits et d'accélérer le temps de résolution.

Compte tenu des résultats obtenus dans les expérimentations (Figure 3), nous avons fait le choix de tester la résolution du problème global en considérant plusieurs configurations de deux groupes de 6 orthophonistes disjoints. En effet, nous avions conclu que plus le nombre d'employés est élevé, plus le gain lié à la mutualisation des orthophonistes est important (Figure 2). La taille limite de 6 orthophonistes est donc un bon compromis entre le gain potentiel et le temps de calcul. Dans notre cas, la résolution du problème consiste donc à résoudre séparément deux sous-problèmes, pour lesquels on obtient les résultats en un temps très court.

Il existe différentes approches pour créer des clusters (Decerle 2018), qui diffèrent soit par la forme de construction des clusters, soit par les indicateurs utilisés. Dans notre cas, nous avons fait le choix de constituer les clusters en regroupant 6 professionnels et leurs patients respectifs dans un sous-ensemble. Les patients d'un orthophoniste sont associés au même cluster afin de garantir l'existence d'au moins une solution, celle où chaque orthophoniste conserve sa patientèle. Le choix du regroupement des professionnels a quant à lui été fait selon deux critères. Premièrement, nous avons essayé de faire en sorte que chaque groupe ait toutes les qualifications possibles portées par ses membres. Deuxièmement, nous avons regroupé les professionnels en utilisant l'heuristique gloutonne du plus proche voisin. Ainsi, selon leurs proximités géographiques nous séparons les deux professionnels les plus éloignés dans deux clusters distincts et nous affectons successivement les autres professionnels à l'un de ces clusters selon le critère du plus proche voisin. Pour mesurer l'intérêt de la mutualisation, nous avons expérimenté plusieurs découpages que nous comparons à l'approche séparée.

La première configuration correspond à un découpage arbitraire du pool en deux groupes (Orthophonistes 1 à 6, et Orthophonistes 7 à 12). Les configurations 2 et 3 correspondent aux ensembles représentés par les 2 cartes de la figure 4. Elles prennent en considération la localisation du domicile de l'employé, avec deux découpages qui diffèrent au niveau de la répartition des orthophonistes localisés dans le centre de la métropole.

Le tableau 4 présente les résultats obtenus suite à la résolution de 3 configurations comprenant deux clusters distincts, avec comme objectif la minimisation du temps de trajet. Nous les comparons au temps de trajet cumulé obtenu en résolvant le problème de tournée en considérant les orthophonistes individuellement sans mixer les patients. Pour chaque décomposition, le problème a pu être résolu à l'optimal, sauf pour le groupe $\mathrm{B}$ de la configuration 2. Le solveur est arrivé à un objectif de 407 après plusieurs heures de calcul, sans avoir prouvé l'optimalité (à 0,25\% de la borne inférieure).

Les résultats montrent tout d'abord que, pour chaque configuration, la résolution du problème de planification sur deux groupes de 6 employés apporte un gain de plus de $20 \%$ par rapport à la résolution mono-employé. L'intérêt de la mutualisation des orthophonistes, du point de vue du temps de trajet pour 


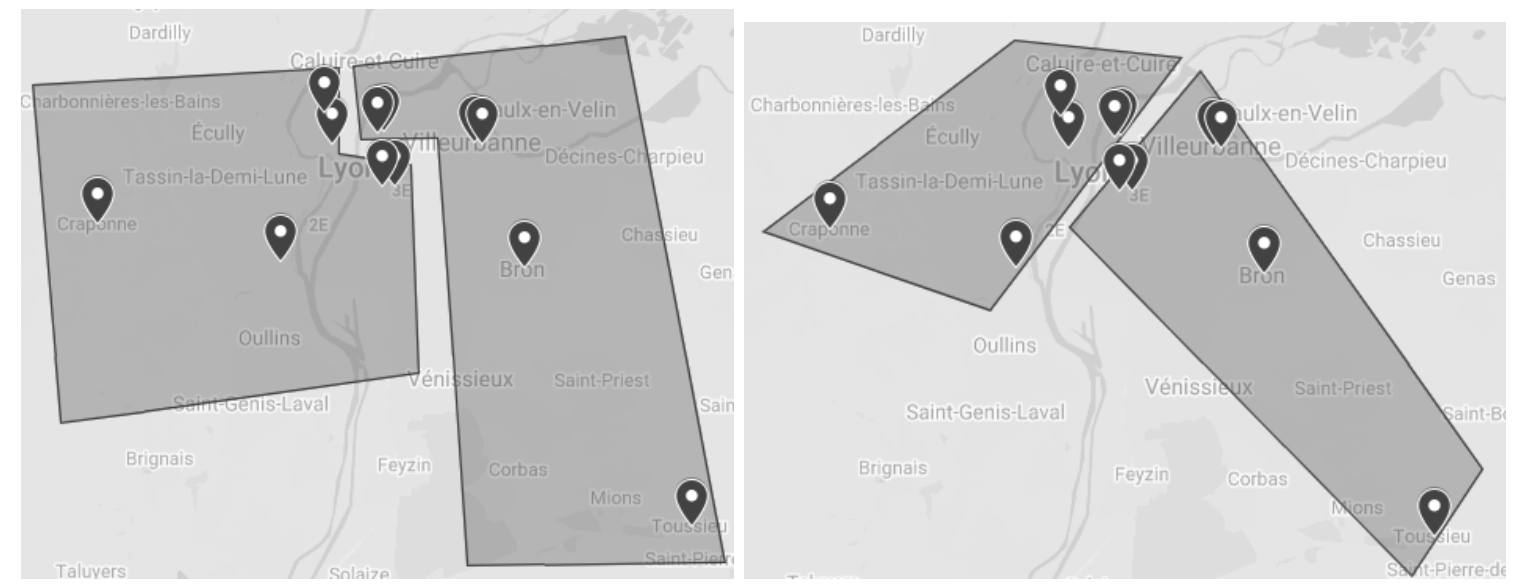

Figure 4.: Carte des deux séparations en 2 clusters d'employés

\begin{tabular}{|c|c|c|c|c|c|c|c|c|}
\hline \multirow[t]{2}{*}{ Config. } & \multirow{2}{*}{\multicolumn{2}{|c|}{$\begin{array}{l}\text { Composition } \\
\text { des groupes } \\
\text { d'employés }\end{array}$}} & \multirow[t]{2}{*}{$\begin{array}{l}\text { Trajet } \\
\text { optimal } \\
\text { (OptTrav) }\end{array}$} & \multirow[t]{2}{*}{$\begin{array}{l}\text { Cumul } \\
\text { OptTrav } \\
\text { Gr. A+B }\end{array}$} & \multicolumn{2}{|c|}{$\begin{array}{l}\text { Cumul OptTrav } \\
\text { Mono-employé }\end{array}$} & \multicolumn{2}{|c|}{$\begin{array}{l}\text { Gain } \\
\text { mutualisation }\end{array}$} \\
\hline & & & & & groupe & total & groupe & total \\
\hline \multirow[t]{2}{*}{1} & Gr.A & 123456 & 392 & \multirow{2}{*}{798} & 598 & \multirow{2}{*}{1086} & $34 \%$ & \multirow[t]{2}{*}{$27 \%$} \\
\hline & Gr. B & 789101112 & 406 & & 488 & & $17 \%$ & \\
\hline \multirow{2}{*}{2} & Gr. A & 25791011 & 454 & \multirow[t]{2}{*}{861} & 507 & \multirow{2}{*}{1086} & $10 \%$ & \multirow{2}{*}{$21 \%$} \\
\hline & Gr. B & 1346812 & 407 & & 579 & & $30 \%$ & \\
\hline \multirow{2}{*}{3} & Gr. A & 245789 & 427 & \multirow{2}{*}{848} & 515 & \multirow{2}{*}{1086} & $17 \%$ & \multirow{2}{*}{$22 \%$} \\
\hline & Gr. B & 136101112 & 421 & & 571 & & $26 \%$ & \\
\hline
\end{tabular}

Tableau 5.7.: Performance des 3 configurations ( $2 \times 6$ employés) testées

réaliser les services aux patients, est ici vérifié.

On remarque que certaines configurations sont meilleures que d'autres, quand on compare le cumul des temps de trajet des groupes A et B. Il est d'ailleurs étonnant que le découpage le plus performant soit celui défini arbitrairement, sans prise en compte du critère géographique. Cela peut s'expliquer par le fait que le critère de dispersion géographique des patients initialement affectés aux orthophonistes n'a pas été considéré dans le découpage. Il est tout à fait possible que des groupes d'orthophonistes organisés de manière arbitraires offrent finalement une meilleure proximité à l'ensemble des patients à servir. Aussi, si l'on veut tirer le meilleur profit de la stratégie de mutualisation, le plus grand soin devra être porté au découpage du pool d'employés en 2 groupes. Cette question pourra aussi s'étendre à la pré-affectation des patients aux clusters. Ainsi, on peut imaginer un algorithme qui opère la répartition des orthophonistes en même temps que celles de patients entre les deux groupes, qui toutefois vérifierait l'existence d'une solution. En effet, une incompatibilité des qualifications des employés et des services demandés par les patients, ou des disponibilités qui ne concordent pas entre employés et patients d'un même groupe, pourrait amener à des problèmes infaisables. 


\section{Conclusion et perspectives}

Nous avons proposé un modèle pour la résolution d'un problème d'affectation et de tournées d'orthophonistes auprès d'enfants en situation de handicap. L'utilisation de la méthode intégrée, dans laquelle on considère un pool d'orthophonistes permet d'économiser à la fois le temps de trajet et le temps d'attente. Cependant, le temps de calcul de la solution optimale devient trop élevé à partir de 6 employés dans le pool. Nous avons donc proposé une démarche de clustering pour surmonter cette contrainte de nombre de variables, en cherchant à séparer le problème en sous-problèmes, de tailles amenant à un temps de résolution raisonnable. Les expérimentations montrent l'intérêt d'une telle démarche.

Concernant l'approche PLNE, une perspective serait d'explorer la possibilité d'équilibrer de la charge de travail entre les orthophonistes. N'ayant pas les mêmes contrats en termes d'heures par semaine, une charge de travail équilibrée entre les employés serait précieuse pour le gestionnaire pour éviter de surcharger ou de sous-charger quelqu'un, et promouvoir l'équité au sein de l'équipe.

Autour de la création de clusters, il serait souhaitable de poursuivre les investigations relatives aux mécanismes de construction des clusters qui impactent la qualité des solutions obtenues. Ainsi, il serait pertinent de baser l'algorithme de clustering sur des considérations autres que la proximité géographique et la compétence, afin d'obtenir de meilleurs résultats. À cette fin, il serait pertinent de tester des méthodes plus poussées comme le k-means ou d'exploiter la structuration hiérarchique patients/ praticiens pour mettre en œuvre un clustering hiérarchique.a Nous pourrions aussi intégrer une mesure de la faisabilité du clustering en prenant par exemple une approche plus itérative où le clustering rebouclerait avec les tournées, permettant ainsi certainement de trouver une solution pour chaque sousproblème.

\section{Bibliographie}

Alves, F., Costa, L., Rocha, A., Pereira, A., Leitão, P., «A multi-objective approach to the optimization of home care visits scheduling », 8th International Conference on Operations Research and Enterprise Systems, ICORES 2019, (2019) : 435-442.

Cissé, M., Yalçindag, S., Kergosien, Y., Sahin, E. Lenté, C., Matta, A., «Operations Research for Health Care OR problems related to Home Health Care : A review of relevant routing and scheduling problems. » Operations Research for Health Care, $\mathrm{n}^{\circ}$ 13-14 (2017) :1-22.

DECERLE, J., Contribution à l'optimisation de la planification des tournées de soins pour l'hospitalisation à domicile, Thèse de doctorat à UTBM Belfort, 2018.

Decerle, J., Grunder, O., Hajuam El Hassani, A., Barakat, O., «A memetic algorithm for multi-objective optimization of the home health care problem. » Swarm and Evolutionary Computation, $\mathrm{n}^{\circ} 44$ (2019) : 712-727.

Demirbilek, M., Branke, J., Strauss, A., 2018. «Dynamically accepting and scheduling patients for home healthcare. » Health Care Management Science, ${ }^{\circ} 22$ (2018):140-15

Di Mascolo, M., Martinez, M., Espinouse, M.-L., «Routing and scheduling in Home Health Care : A Literature Survey and Bibliometric Analysis. » Computers Industrial Engineering, $\mathrm{n}^{\circ} 158$ (2021).

Grenouilleau, F., Legrain, A., Lahrichi, N., Rousseau, L.M.. «A set partitioning heuristic for the home health care routing and scheduling problem. » European Journal of Operational Research, n²75 (2019) : 295-303.

Grieco, L., Utley, M., Crowe, S., «Operational research applied to decisions in home health care : A systematic literature review. » Journal of the Operational Research Society, 2020

Heching, A., Hooker, J.N., Kimura, R., « A Logic-Based Benders Approach to Home Healthcare Delivery. » Transportation Science $\mathrm{n}^{\circ} 53$ (2019) : 510-522.

Martinez, C., Espinouse, M.-L., Di Mascolo, M., «Continuity of care in home services : a client-centered heuristic for the home health care routing and scheduling problem. » 5th International Conference on Control, Decision and Information Technologies (CoDIT) (2018) :1045-1050. 
Tellez O., Vercraene, S., Lehuédé, F., Péton, O., Monteiro, T., 2020. « The time-consistent dial-a-ride problem. »(2020)<hal-02460670> 